\title{
GENERALIZED HADAMARD'S INEQUALITIES BASED ON GENERAL EULER 4-POINT FORMULAE
}

\author{
M. KLARIČIĆ BAKULA ${ }^{\approx 1}$ and J. PEČARIĆ ${ }^{2}$
}

(Received 19 April, 2006)

\begin{abstract}
We present a general closed 4-point quadrature rule based on Euler-type identities. We use this rule to prove a generalization of Hadamard's inequalities for $(2 r)$-convex functions $(r \geq 1)$.

2000 Mathematics subject classification: primary 26D15, 26D20.

Keywords and phrases: Euler formulae, quadrature rules, Hadamard's inequalities, convex functions.
\end{abstract}

\section{Introduction}

Let $f$ be a convex function on $[a, b] \subset \mathbb{R}, a \neq b$. The following double inequality:

$$
f\left(\frac{a+b}{2}\right) \leq \frac{1}{b-a} \int_{a}^{b} f(x) d x \leq \frac{f(a)+f(b)}{2}
$$

is known in the literature as Hadamard's inequalities (see for example [10, page 137]) for convex functions.

Hadamard's inequalities can be generalized in the following way.

THEOREM 1.1. Let $f:[a, b] \rightarrow \mathbb{R}$ be a convex function. Then for every $x \in$ $[a,(a+b) / 2]$

$$
\begin{aligned}
& \frac{f(a)+f(b)}{2}-\frac{1}{b-a} \int_{a}^{b} f(t) d t \\
& \geq \frac{1}{b-a} \int_{a}^{b} f(t) d t-\frac{f(x)+f(a+b-x)}{2}
\end{aligned}
$$

\footnotetext{
${ }^{1}$ Department of Mathematics, Faculty of Natural Sciences, Mathematics and Education, University of Split, Teslina 12, 21000 Split, Croatia; e-mail: milica@pmfst.hr.

${ }^{2}$ Faculty of Textile Technology, University of Zagreb, Pierottijeva 6, 10000 Zagreb, Croatia; e-mail: pecaric@hazu.hr.
}

(C) Australian Mathematical Society 2007, Serial-fee code 1446-1811/07 
and for every $x \in[(3 a+b) / 4,(a+b) / 2]$

$$
\frac{1}{b-a} \int_{a}^{b} f(t) d t-\frac{f(x)+f(a+b-x)}{2} \geq 0 .
$$

PROOF. Let $x \in[a,(a+b) / 2]$. Since $f$ is convex on $[a, b]$, the right-hand side of (1.1) gives

$$
\begin{aligned}
\frac{1}{b-a} & \int_{a}^{b} f(t) d t \\
= & \frac{1}{b-a}\left[\int_{a}^{x} f(t) d t+\int_{x}^{a+b-x} f(t) d t+\int_{a+b-x}^{b} f(t) d t\right] \\
\leq & \frac{1}{b-a}\left[(x-a) \frac{f(a)+f(x)}{2}+(a+b-2 x) \frac{f(x)+f(a+b-x)}{2}\right. \\
& \left.+(x-a) \frac{f(a+b-x)+f(b)}{2}\right] \\
= & \frac{1}{2}\left[\frac{x-a}{b-a}(f(a)+f(b))+\frac{b-x}{b-a}(f(x)+f(a+b-x))\right]
\end{aligned}
$$

Since $f$ is convex on $[a, b]$, for any $h>0$ and $x_{1}, x_{2} \in[a, b]$ such that $x_{1} \leq x_{2}$ we have (see, for example, [11, pages 5,6$]$ )

$$
f\left(x_{1}+h\right)-f\left(x_{1}\right) \leq f\left(x_{2}+h\right)-f\left(x_{2}\right) .
$$

Consider now $x \in[a,(a+b) / 2]$. If we apply (1.5) on $h=x-a, x_{1}=a$ and $x_{2}=a+b-x$, we obtain

$$
f(x)-f(a) \leq f(b)-f(a+b-x) .
$$

For $x \in[a,(a+b) / 2]$ we have $a+b-2 x \geq 0$, so for such $x$ the inequality (1.6) can be rewritten as

that is,

$$
(a+b-2 x) \frac{f(x)-f(a)}{b-a} \leq(a+b-2 x) \frac{f(b)-f(a+b-x)}{b-a},
$$

$$
(a+b-2 x) \frac{f(x)-f(a)}{b-a}+(2 x-a-b) \frac{f(b)-f(a+b-x)}{b-a} \leq 0 .
$$

From this, a simple calculation gives us

$$
\begin{aligned}
& \frac{2(x-a)}{b-a}[f(a)+f(b)]+\frac{2(b-x)}{b-a}[f(x)+f(a+b-x)] \\
& \leq f(a)+f(b)+f(x)+f(a+b-x) .
\end{aligned}
$$


Combining (1.4) and (1.7) we obtain

$$
\frac{1}{b-a} \int_{a}^{b} f(t) d t \leq \frac{f(a)+\dot{f}(b)+f(x)+f(a+b-x)}{4}
$$

from which we get

$$
\frac{f(a)+f(b)}{2}-\frac{1}{b-a} \int_{a}^{b} f(t) d t \geq \frac{1}{b-a} \int_{a}^{b} f(t) d t-\frac{f(x)+f(a+b-x)}{2},
$$

and this completes the proof of (1.2).

Now let $x \in[(3 a+b) / 4,(a+b) / 2]$. Since $f$ is convex on $[a, b]$, the left-hand side of (1.1) gives

$$
\begin{aligned}
\frac{1}{b-a} \int_{a}^{b} f(t) d t & =\frac{1}{b-a}\left[\int_{a}^{(a+b) / 2} f(t) d t+\int_{(a+b) / 2}^{b} f(t) d t\right] \\
& \geq \frac{1}{b-a}\left[\frac{b-a}{2} f\left(\frac{3 a+b}{4}\right)+\frac{b-a}{2} f\left(\frac{a+3 b}{4}\right)\right] \\
& =\frac{1}{2}\left[f\left(\frac{3 a+b}{4}\right)+f\left(\frac{a+3 b}{4}\right)\right]
\end{aligned}
$$

If we apply (1.5) again on $h=(4 x-3 a-b) / 4, x_{1}=(3 a+b) / 4$ and $x_{2}=a+b-x$, we obtain

$$
f(x)-f\left(\frac{3 a+b}{4}\right) \leq f\left(\frac{a+3 b}{4}\right)-f(a+b-x)
$$

that is,

$$
f(x)+f(a+b-x) \leq f\left(\frac{a+3 b}{4}\right)+f\left(\frac{3 a+b}{4}\right) .
$$

Combining (1.9) with (1.8) we obtain

$$
\frac{1}{b-a} \int_{a}^{b} f(t) d t \geq \frac{f(x)+f(a+b-x)}{2}
$$

so the inequality (1.3) is proved.

REMARK 1. If in (1.2) and (1.3) we let $x=(a+b) / 2$, we obtain

$$
\frac{f(a)+f(b)}{2}-\frac{1}{b-a} \int_{a}^{b} f(t) d t \geq \frac{1}{b-a} \int_{a}^{b} f(t) d t-f\left(\frac{a+b}{2}\right) \geq 0,
$$

which is one of Bullen's results from [3]. His result was generalized for $(2 r)$-convex functions $(r \in \mathbb{N})$ in [6]. 
The goal of this paper is to obtain a variant of Inequalities (1.2) and (1.3) for (2r)-convex functions $(r \in \mathbb{N}$ ). To achieve this goal we will construct a general closed 4-point rule based on Euler-type identities established in [4].

We recall that a function $f:[a, b] \rightarrow \mathbb{R}$ is said to be $n$-convex on $[a, b]$ for some $n \geq 0$ if for any choice of $n+1$ points $x_{0}, \ldots, x_{n}$ from $[a, b]$ we have $\left[x_{0}, \ldots, x_{n}\right] f \geq 0$, where $\left[x_{0}, \ldots, x_{n}\right] f$ is the $n$-th order divided difference of $f$. If $f$ is $n$-convex, then $f^{(n-2)}$ exists and is an convex function in the ordinary sense. Also, if $f^{(n)}$ exists, then $f$ is $n$-convex if and only if $f^{(n)} \geq 0$. For more details see for example [10].

It should be noted that each continuous $n$-convex function on $[a, b]$ is the uniform limit of a sequence of the corresponding Bernstein's polynomials (see, for example, [10, page 293]). Bernstein polynomials of any continuous $n$-convex function are also $n$-convex functions, so when stating our results for a continuous $(2 r)$-convex function $f$ without any loss in generality we may assume that $f^{(2 r)}$ exists and is continuous. Actually, our results are valid for any continuous $(2 r)$-convex function $f$.

In Section 2 we present a general closed 4-point quadrature rule based on the extended Euler formulae and we also give two estimations of the remainder. In Section 3 we use the obtained results to prove a generalization of Hadamard's inequalities for (2r)-convex functions $(r \in \mathbb{N})$.

\section{General closed 4-point quadrature rule}

In the paper [4] two identities, named the extended Euler formulae, have been proved. They are given in the following theorem.

THEOREM A. Let $f:[a, b] \rightarrow \mathbb{R}$ be such that $f^{(n-1)}$ is a continuous function of bounded variation on $[a, b]$ for some $n \in \mathbb{N}$. Then for every $x \in[a, b]$

$$
f(x)=\frac{1}{b-a} \int_{a}^{b} f(t) d t+T_{n}(x)+R_{n}^{1}(x)
$$

and

$$
f(x)=\frac{1}{b-a} \int_{a}^{b} f(t) d t+T_{n-1}(x)+R_{n}^{2}(x),
$$

where

$$
\begin{aligned}
& T_{m}(x)=\sum_{k=1}^{m} \frac{(b-a)^{k-1}}{k !} B_{k}\left(\frac{x-a}{b-a}\right)\left[f^{(k-1)}(b)-f^{(k-1)}(a)\right], \\
& R_{n}^{1}(x)=-\frac{(b-a)^{n-1}}{n !} \int_{[a, b]} B_{n}^{*}\left(\frac{x-t}{b-a}\right) d f^{(n-1)}(t),
\end{aligned}
$$


and

$$
R_{n}^{2}(x)=-\frac{(b-a)^{n-1}}{n !} \int_{[a, b]}\left[B_{n}^{*}\left(\frac{x-t}{b-a}\right)-B_{n}\left(\frac{x-a}{b-a}\right)\right] d f^{(n-1)}(t) .
$$

Here, as in the rest of the paper, the functions $B_{k}(\cdot)(k \geq 0)$ are the Bernoulli polynomials, $B_{k}$ are the Bernoulli numbers and $B_{k}^{*}(\cdot)$ are periodic functions of period one, related to the Bernoulli polynomials as

$$
\begin{aligned}
B_{k}^{*}(x) & =B_{k}(x), & & 0 \leq x<1, \\
B_{k}^{*}(x+1) & =B_{k}^{*}(x), & & x \in \mathbb{R} .
\end{aligned}
$$

In this paper we write $\int_{[a, b]} g(t) d \varphi(t)$ to denote the Riemann-Stieltjes integral of a function $g:[a, b] \rightarrow \mathbb{R}$ with respect to a continuous function $\varphi:[a, b] \rightarrow \mathbb{R}$ of bounded variation, and we write $\int_{a}^{b} g(t) d t$ for the Riemann integral.

To make reading easier, let us recall some of the properties of the Bernoulli polynomials (see, for example, $[1,23.1]$ or [2]). The Bernoulli polynomials are uniquely determined by the following identities:

$$
\begin{aligned}
B_{0}(x) & =1, \quad x \in \mathbb{R}, \\
B_{k}^{\prime}(x) & =k B_{k-1}(x), \quad k \geq 1, \\
B_{k}(x+1)-B_{k}(x) & =k x^{k-1}, \quad k \geq 0 .
\end{aligned}
$$

From that we have $B_{1}(x)=x-1 / 2, B_{2}(x)=x^{2}-x+1 / 6, B_{3}(x)=x^{3}-3 x^{2} / 2+x / 2$, so that $B_{0}^{*}$ and $B_{1}^{*}$ are discontinuous functions with jumps of -1 at each integer. Also, it follows that $B_{k}(1)=B_{k}(0)=B_{k}$ for $k \geq 2$, so that $B_{k}^{*}$ are continuous functions for $k \geq 2$. From this we get $\left(B_{k}^{*}\right)^{\prime}(x)=k B_{k-1}^{*}(x), k \geq 1$, for every $t \in \mathbb{R}$ if $k \geq 3$ and for every $t \in \mathbb{R} \backslash \mathbb{Z}$ if $k=1,2$.

Here we list some of the properties of the Bernoulli polynomials which will be used in this paper (see, for example, [1] or [2]):

$$
\begin{aligned}
B_{k}(1-x) & =(-1)^{k} B_{k}(x), \quad n \geq 0, \quad x \in \mathbb{R}, \\
B_{k}(1 / 2) & =-\left(1-2^{1-k}\right) B_{k}, \quad n \geq 0, \\
B_{2 k-1}(1 / 2) & =B_{2 k-1}=0, \quad k \geq 1, \\
B_{k}(0) & =B_{k}(1), \quad k \geq 2, \\
(-1)^{k} B_{2 k-1}(x) & >0, \quad k \geq 1, \quad x \in(0,1 / 2), \\
(-1)^{k-1} B_{2 k} & >0, \quad r \geq 0 .
\end{aligned}
$$

For $k \geq 1$ and fixed $x \in[a,(a+b) / 2]$ we define functions $G_{k}^{x}$ and $F_{k}^{x}$ as

$$
\begin{aligned}
G_{k}^{x}(t) & =B_{k}^{*}\left(\frac{x-t}{b-a}\right)+B_{k}^{*}\left(\frac{a+b-x-t}{b-a}\right)+B_{k}^{*}\left(\frac{a-t}{b-a}\right)+B_{k}^{*}\left(\frac{b-t}{b-a}\right) \\
& =B_{k}^{*}\left(\frac{x-t}{b-a}\right)+B_{k}^{*}\left(\frac{a+b-x-t}{b-a}\right)+2 B_{k}^{*}\left(\frac{a-t}{b-a}\right)
\end{aligned}
$$


and $F_{k}^{x}(t)=G_{k}^{x}(t)-\widetilde{B}_{k}^{x}$, for all $t \in \mathbb{R}$, where

$$
\begin{aligned}
\widetilde{B}_{k}^{x} & =B_{k}\left(\frac{x-a}{b-a}\right)+B_{k}\left(\frac{b-x}{b-a}\right)+B_{k}(0)+B_{k}(1) \\
& =\left[1+(-1)^{k}\right]\left[B_{k}\left(\frac{x-a}{b-a}\right)+B_{k}\right] .
\end{aligned}
$$

Of course, if $k \geq 2$ we have $\widetilde{B}_{k}^{x}=\left[1+(-1)^{k}\right] B_{k}((x-a) /(b-a))+2 B_{k}$. Using the properties of the Bernoulli polynomials which were mentioned in the introduction, we can easily see that for any $x \in[a,(a+b) / 2]$

$$
\begin{aligned}
& \widetilde{B}_{k}^{x}=G_{k}^{x}(a), \quad k \geq 2, \quad \widetilde{B}_{2 r-1}^{x}=0, \quad r \geq 1, \\
& \widetilde{B}_{2 r}^{x}=2\left[B_{2 r}\left(\frac{x-a}{b-a}\right)+B_{2 r}\right], \quad r \geq 1, \\
& F_{2 i-1}^{x}(t)=G_{2 t-1}^{x}(t), \quad i \geq 1, \\
& F_{2 r}^{x}(t)=G_{2 r}^{x}(t)-2\left[B_{2 r}\left(\frac{x-a}{b-a}\right)+B_{2 r}\right], \quad r \geq 1, \\
& F_{k}^{x}(a)=F_{k}^{x}(b)=0, \quad k \geq 1, \\
& G_{k}^{x}(a)=G_{k}^{x}(b)=\left[1+(-1)^{k}\right] B_{k}\left(\frac{x-a}{b-a}\right)+2 B_{k}, \quad k \geq 1 .
\end{aligned}
$$

We can also easily check that for all $r \geq 1$

and

$$
F_{2 r-1}^{x}\left(\frac{a+b}{2}\right)=G_{2 r-1}^{x}\left(\frac{a+b}{2}\right)=0
$$

$$
\begin{aligned}
G_{2 r}^{x}\left(\frac{a+b}{2}\right) & =2 B_{2 r}\left(\frac{1}{2}-\frac{x-a}{b-a}\right)+2 B_{2 r}\left(\frac{1}{2}\right) \\
F_{2 r}^{x}\left(\frac{a+b}{2}\right) & =G_{2 r}^{x}\left(\frac{a+b}{2}\right)-\widetilde{B}_{2 r}^{x} \\
& =2\left[B_{2 r}\left(\frac{1}{2}-\frac{x-a}{b-a}\right)-B_{2 r}\left(\frac{x-a}{b-a}\right)+B_{2 r}\left(\frac{1}{2}\right)-B_{2 r}\right] \\
& =2\left[B_{2 r}\left(\frac{1}{2}-\frac{x-a}{b-a}\right)-B_{2 r}\left(\frac{x-a}{b-a}\right)+2\left(2^{-2 r}-1\right) B_{2 r}\right] .
\end{aligned}
$$

Now let $f:[a, b] \rightarrow \mathbb{R}$ be such that $f^{(n-1)}$ exists on $[a, b]$ for some $n \geq 1$. We introduce the following notation for each $x \in[a,(a+b) / 2]$ :

$$
D(x)=[f(x)+f(a+b-x)+f(a)+f(b)] / 4 .
$$


Furthermore, we define

$$
\begin{aligned}
& \widetilde{T}_{0}(x)=0 \\
& \widetilde{T}_{m}(x)=\frac{1}{4}\left[T_{m}(x)+T_{m}(a+b-x)+T_{m}(a)+T_{m}(b)\right], \quad 1 \leq m \leq n
\end{aligned}
$$

where $T_{m}$ is given by (2.3). It can be easily checked that

$$
\widetilde{T}_{m}(x)=\frac{1}{4} \sum_{k=1}^{m} \frac{(b-a)^{k-1}}{k !} \widetilde{B}_{k}^{x}\left[f^{(k-1)}(b)-f^{(k-1)}(a)\right] .
$$

For further use we will denote

$$
\widetilde{T}_{m}^{\nu}(x)=\frac{T_{m}(x)+T_{m}(a+b-x)}{2} \quad \text { and } \quad \widetilde{T}_{m}^{F}=\frac{T_{m}(a)+T_{m}(b)}{2} .
$$

Obviously, $\widetilde{T}_{m}(x)=\left(\widetilde{T}_{m}^{V}(x)+\widetilde{T}_{m}^{F}\right) / 2$.

THEOREM 2.1. Let $f:[a, b] \rightarrow \mathbb{R}, a<b$, be such that for some $n \in \mathbb{N}$, the derivative $f^{(n-1)}$ is a continuous function of bounded variation on $[a, b]$. Then for every $x \in[a, b]$

$$
\begin{aligned}
& \frac{1}{b-a} \int_{a}^{b} f(t) d t=D(x)-\widetilde{T}_{n}(x)+\widetilde{R}_{n}^{1}(x) \text { and } \\
& \frac{1}{b-a} \int_{a}^{b} f(t) d t=D(x)-\widetilde{T}_{n-1}(x)+\widetilde{R}_{n}^{2}(x),
\end{aligned}
$$

where

$$
\begin{aligned}
& \widetilde{R}_{n}^{!}(x)=\frac{(b-a)^{n-1}}{4 n !} \int_{[a, b]} G_{n}^{x}(t) d f^{(n-1)}(t) \text { and } \\
& \widetilde{R}_{n}^{2}(x)=\frac{(b-a)^{n-1}}{4 n !} \int_{[a, b]} F_{n}^{x}(t) d f^{(n-1)}(t) .
\end{aligned}
$$

PROOF. Put $x \equiv x, a+b-x, a, b$ in the formula (2.1) to get four new formulae. Then multiply these formulae by $1 / 4$ and add. The result is (2.4), and (2.5) is obtained from (2.2) by the same procedure.

REMARK 2. If in Theorem 2.1 we choose $x=a$ we obtain the Euler trapezoidal rule [5], and if we choose $x=(a+b) / 2$ we obtain the Euler bitrapezoidal rule [6].

Our next goal is to give an estimation of the remainder $\widetilde{R}_{n}^{2}(x)$. For the sake of simplicity we will temporarily introduce two new variables:

$$
\xi=\frac{x-a}{b-a} \text { and } s=\frac{t-a}{b-a} .
$$


It can be easily seen that for $x, t \in[a, b]$ we have $\xi, s \in[0,1]$. Using direct calculations, for each $\xi \in[0,1 / 2]$ we obtain

$$
\begin{aligned}
& G_{1}^{\xi}(s)=F_{1}^{\xi}(s)= \begin{cases}-4 s+1, & 0 \leq s \leq \xi, \\
-4 s+2, & \xi<s \leq 1-\xi, \\
-4 s+3, & 1-\xi<s<1,\end{cases} \\
& G_{2}^{\xi}(s)= \begin{cases}4 s^{2}-2 s+2 \xi^{2}-2 \xi+2 / 3, & 0 \leq s \leq \xi, \\
4 s^{2}-4 s+2 \xi^{2}+2 / 3, & \xi<s \leq 1-\xi, \\
4 s^{2}-6 s+2 \xi^{2}-2 \xi+8 / 3, & 1-\xi<s<1,\end{cases} \\
& F_{2}^{\xi}(s)= \begin{cases}4 s^{2}-2 s, & 0 \leq s \leq \xi, \\
4 s^{2}-4 s+2 \xi, & \xi<s \leq 1-\xi, \\
4 s^{2}-6 s+2, & 1-\xi<s<1,\end{cases} \\
& G_{3}^{\xi}(s)= \begin{cases}-4 s^{3}+3 s^{2}-2 s\left(3 \xi^{2}-3 \xi+1\right), & 0 \leq s \leq \xi, \\
-4 s^{3}+6 s^{2}-2 s\left(3 \xi^{2}+1\right)+3 \xi^{2}, & \xi<s \leq 1-\xi, \\
-4 s^{3}+9 s^{2}-2 s\left(3 \xi^{2}-3 \xi+4\right)+6 \xi^{2}-6 \xi+3, & 1-\xi<s<1,\end{cases} \\
& =F_{3}^{\xi}(s) .
\end{aligned}
$$

Next we present some properties of the functions $G_{k}^{\xi}$ and $F_{k}^{\xi}$. First we prove that the functions $G_{k}^{\xi}$ and $F_{k}^{\xi}$ are symmetric for even $k$ and skew-symmetric for odd $k$ with respect to $1 / 2$.

LEMMA 2.2. Let $\xi \in[0,1 / 2]$ be fixed. For $k \geq 2$ and $s \in[0,1]$, we have

$$
G_{k}^{\xi}(1-s)=(-1)^{k} G_{k}^{\xi}(s) \text { and } F_{k}^{\xi}(1-s)=(-1)^{k} F_{k}^{\xi}(s) .
$$

PROOF. As stated at the beginning of this section, for $k \geq 2$ and $s \in[0,1]$, we have

$$
\begin{aligned}
G_{k}^{\xi}(1 & -s) \\
& =B_{k}^{*}(\xi-1+s)+B_{k}^{*}(-\xi+s)+2 B_{k}^{*}(s) \\
& = \begin{cases}B_{k}(\xi+s)+B_{k}(1-\xi+s)+2 B_{k}(s), & 0 \leq s \leq \xi, \\
B_{k}(\xi+s)+B_{k}(-\xi+s)+2 B_{k}(s), & \xi<s \leq 1-\xi, \\
B_{k}(\xi-1+s)+B_{k}(-\xi+s)+2 B_{k}(s), & 1-\xi<s \leq 1,\end{cases} \\
& =(-1)^{k} \begin{cases}B_{k}(1-\xi-s)+B_{k}(\xi-s)+2 B_{k}(1-s), & 0 \leq s \leq \xi, \\
B_{k}(1-\xi-s)+B_{k}(1+\xi-s)+2 B_{k}(1-s), & \xi<s \leq 1-\xi, \\
B_{k}(2-\xi-s)+B_{k}(1+\xi-s)+2 B_{k}(1-s), & 1-\xi<s \leq 1,\end{cases} \\
& =(-1)^{k} G_{k}^{\xi}(s),
\end{aligned}
$$


which proves the first identity. Further, we know that $F_{k}^{\xi}(s)=G_{k}^{\xi}(s)-G_{k}^{\xi}(0)$. If $k=2 i-1, i \geq 2$, then $G_{2 i-1}^{\xi}(0)=G_{2 i-1}^{\xi}(1)=0$, so we immediately have

$$
F_{2 \imath-1}^{\xi}(1-s)=G_{2 t-1}^{\xi}(1-s)=(-1)^{2 t-1} G_{2 \imath-1}^{\xi}(s)=(-1)^{2 \imath-1} F_{2 \imath-1}^{\xi}(s)
$$

On the other hand, if $k=2 i, i \geq 1$, then $(-1)^{2 i}=1$, so we obtain

$$
\begin{aligned}
F_{2 i}^{\xi}(1-s) & =G_{2 i}^{\xi}(1-s)+G_{2 i}^{\xi}(0) \\
& =(-1)^{2 i} G_{2 i}^{\xi}(s)+(-1)^{2 i} G_{2 i}^{\xi}(0)=(-1)^{2 i} F_{2 i}^{\xi}(s),
\end{aligned}
$$

and this proves the second identity.

REMARK 3. It is obvious that analogous assertions hold true for the functions $G_{k}^{x}$ and $F_{k}^{x}, k \geq 2$. In other words, if $x \in[a,(a+b) / 2]$ and $t \in[a, b]$ we have

$$
G_{k}^{x}(b-t)=(-1)^{k} G_{k}^{x}(t) \quad \text { and } \quad F_{k}^{x}(b-t)=(-1)^{k} F_{k}^{x}(t) .
$$

LEMMA 2.3. If $\xi \in[0,1 / 2-1 /(4 \sqrt{6}))$, then for all $s \in(0,1 / 2), G_{3}^{\xi}(s)<0$. Also

$$
\begin{aligned}
G_{3}^{1 / 2-1 /(4 \sqrt{6})}(s)<0, & s \in(0,1 / 2) \backslash\{3 / 8\} \\
G_{3}^{1 / 2}(s)<0, & s \in(0,1 / 4) \\
G_{3}^{1 / 2}(s)>0, & s \in(1 / 4,1 / 2) .
\end{aligned}
$$

ProOF. For the sake of simplicity we will denote

$$
\begin{aligned}
G_{3}^{\xi}(s) & = \begin{cases}-4 s^{3}+3 s^{2}-2 s\left(3 \xi^{2}-3 \xi+1\right), & 0 \leq s \leq \xi, \\
-4 s^{3}+6 s^{2}-2 s\left(3 \xi^{2}+1\right)+3 \xi^{2}, & \xi<s \leq 1-\xi \\
-4 s^{3}+9 s^{2}-2 s\left(3 \xi^{2}-3 \xi+4\right)+6 \xi^{2}-6 \xi+3, & 1-\xi<s<1,\end{cases} \\
& = \begin{cases}H_{1}^{\xi}(s), & 0 \leq s \leq \xi \\
H_{2}^{\xi}(s), & \xi<s \leq 1-\xi \\
H_{3}^{\xi}(s), & 1-\xi<s \leq 1 .\end{cases}
\end{aligned}
$$

If we write $H_{1}^{\xi}(s)$ as $H_{1}^{\xi}(s)=s\left[-4 s^{2}+3 s-2\left(3 \xi^{2}-3 \xi+1\right)\right]$, we can see that $H_{1}^{\xi}(0)=0$ and that $H_{1}^{\xi}(\xi)=\xi\left(-10 \xi^{2}+9 \xi-2\right)$, so if for a given $\xi \in[0,1 / 2]$ the number $-10 \xi^{2}+9 \xi-2$ is negative it means that the joining point $\left(\xi, H_{1}^{\xi}(\xi)\right)=$ $\left(\xi, H_{2}^{\xi}(\xi)\right)$ is under the $x$-axis. This will be true for $\xi \in[0,2 / 5)$. The sign of $H_{1}^{\xi}(s)$ is determined by the sign of the function $y(s)=-4 s^{2}+3 s-2\left(3 \xi^{2}-3 \xi+1\right)$. This function will have zeros $s_{1}=3 / 8-(\sqrt{D}) / 8$ and $s_{2}=3 / 8+(\sqrt{D}) / 8$ if $D=-96 \xi^{2}+96 \xi-23 \geq 0$, that is, if $\xi \in[1 / 2-1 /(4 \sqrt{6}), 1 / 2]$. Furthermore, $y(0)=-2\left(3 \xi^{2}-3 \xi+1\right)<0$ which means that (if they exist) both zeros $s_{1}$ and 
$s_{2}$ are positive. Of course, if $\xi=1 / 2-1 /(4 \sqrt{6})$ the function $y$ has only one zero $s=3 / 8$. We want to know if it is possible for $\xi \in(1 / 2-1 /(4 \sqrt{6}), 2 / 5)$ to have $\xi<s_{1}$ (because this will imply that $H_{1}^{\xi}(s)<0$ for all $\left.0 \leq s \leq \xi\right)$. This in fact is not possible because if $\xi<s_{1}$ then we have $\xi<3 / 8$, and $3 / 8<1 / 2-1 /(4 \sqrt{6})$. This means that $H_{1}^{\xi}(s) \leq 0$ for all $s \in(0, \xi)$ can be true only if $D \leq 0$, and this will be true for $\xi \in[0,1 / 2-1 /(4 \sqrt{6})] \subset[0,2 / 5)$.

Now we must check $H_{2}^{\xi}$ for such $\xi$. If $\xi<s \leq 1 / 2$ we have

$$
\begin{aligned}
& H_{2}^{\xi \prime}(s)=-12 s^{2}+12 s-2\left(3 \xi^{2}+1\right), \\
& H_{2}^{\xi \prime \prime}(s)=-24 s+12=12(1-2 s)>0,
\end{aligned}
$$

which means that $H_{2}^{\xi}$ is convex for any choice of such $\xi$. Since $H_{2}^{\xi}(\xi)<0$ and $H_{2}^{\xi}(1 / 2)=0$, we can deduce that $H_{2}^{\xi}(s)<0$ for all $s \in(\xi, 1 / 2)$. This means that if $\xi \in[0,1 / 2-1 /(4 \sqrt{6}))$, then $G_{3}^{\xi}(s)<0, s \in(0,1 / 2)$, and for $\xi=1 / 2-1 /(4 \sqrt{6})$ we have $G_{3}^{\xi}(s)<0, s \in(0,1 / 2) \backslash\{3 / 8\}$.

On the other hand, if $\xi \in(2 / 5,1 / 2]$ the joining point $\left(\xi, H_{1}^{\xi}(\xi)\right)=\left(\xi, H_{2}^{\xi}(\xi)\right)$ is above the $x$-axis, and we want $H_{1}^{\xi}(s)$ to be positive for all $s \in(0, \xi)$. This, of course, cannot be true because $(2 / 5,1 / 2] \subset(1 / 2-1 /(4 \sqrt{6}), 1 / 2]$, which means that $H_{1}^{\xi}$ surely has a zero $s_{1}<3 / 8<2 / 5<\xi$.

And in the end, we must separately investigate $G_{3}^{1 / 2}$ because at this special point $\xi=1 / 2$ the function $G_{3}^{\xi}$ has only one branch for $s \in[0,1 / 2]$, that is, we have

$$
G_{3}^{1 / 2}(s)=s\left(-4 s^{2}+3 s-1 / 2\right), \quad s \in[0,1 / 2] .
$$

We can easily see that $G_{3}^{1 / 2}(s)<0, s \in(0,1 / 4)$ and $G_{3}^{1 / 2}(s)>0, s \in(1 / 4,1 / 2)$.

Of course, from the above results we have $G_{3}^{x}(t)<0, t \in(a,(a+b) / 2)$ for any $x \in[a,(a+b) / 2-(b-a) /(4 \sqrt{6}))$, and also

$$
\begin{aligned}
G_{3}^{(a+b) / 2-(b-a) /(4 \sqrt{6})}(s)<0, & s \in(a,(a+b) / 2) \backslash\{(5 a+3 b) / 8\}, \\
G_{3}^{(a+b) / 2}(t)<0, & t \in(a,(a+b) / 4), \\
G_{3}^{(a+b) / 2}(t)>0, & t \in((3 a+b) / 4,(a+b) / 2) .
\end{aligned}
$$

LEMMA 2.4. For $r \geq 2$ and $x \in[a,(a+b) / 2-(b-a) /(4 \sqrt{6}))$, the function $G_{2 r-1}^{x}$ has no zeros in the interval $(a,(a+b) / 2)$. The sign of this function is determined by

$$
(-1)^{r-1} G_{2 r-1}^{x}(t)>0, \quad t \in(a,(a+b) / 2) .
$$

Also,

$$
\begin{array}{rlrl}
(-1)^{r-1} G_{2 r-1}^{(a+b) / 2-(b-a) /(4 \sqrt{6})}(t) & >0, & t \in(a,(a+b) / 2) \backslash\{(5 a+3 b) / 8\} \\
(-1)^{r-1} G_{2 r-1}^{(a+b) / 2}(t) & >0, & t \in(a,(3 a+b) / 4), \\
(-1)^{r-1} G_{2 r-1}^{(a+b) / 2}(t)<0, & t \in((3 a+b) / 4,(a+b) / 2) .
\end{array}
$$


PROOF. Let $x \in[a,(a+b) / 2-(b-a) /(4 \sqrt{6}))$. If $r=2$, then the assertion follows from Lemma 2.3. Assume now that $r \geq 3$. In that case we have $2 r-1 \geq 5$ and the function $G_{2 r-1}^{x}$ is continuous and at least twice differentiable. We know that

$$
\begin{aligned}
\left(G_{2 r-1}^{x}\right)^{\prime}(t) & =-\frac{2 r-1}{b-a} G_{2 r-2}^{x}(t), \\
\left(G_{2 r-1}^{x}\right)^{\prime \prime}(t) & =\frac{(2 r-1)(2 r-2)}{(b-a)^{2}} G_{2 r-3}^{x}(t),
\end{aligned}
$$

and that $G_{2 r-1}^{x}(a)=G_{2 r-1}^{x}((a+b) / 2)=0$.

Suppose that $G_{2 r-1}^{x}$ has another zero $\alpha \in(a,(a+b) / 2)$. Then inside each of the intervals $(a, \alpha)$ and $(\alpha,(a+b) / 2)$ the derivative $\left(G_{2 r-1}^{x}\right)^{\prime}$ must have at least one zero, say $\beta_{1} \in(a, \alpha)$ and $\beta_{2} \in(\alpha,(a+b) / 2)$. Therefore, the second derivative $\left(G_{2 r-1}^{x}\right)^{\prime \prime}$ must have at least one zero inside the interval $\left(\beta_{1}, \beta_{2}\right) \subset(a,(a+b) / 2)$. Thus, from the assumption that $G_{2 r-1}^{x}$ has a zero inside the interval $(a,(a+b) / 2)$ it follows that $G_{2 r-3}^{x}$ also has a zero inside the interval $(a,(a+b) / 2)$. From this we could deduce that the function $G_{3}^{x}$ also has a zero inside the interval $(a,(a+b) / 2)$ which is not true. Thus $G_{2 r-1}^{r}$ cannot have a zero inside the interval $(a,(a+b) / 2)$. Furthermore, if $G_{2 r-3}^{x}(t)>0$ for $t \in(a,(a+b) / 2)$, then from (2.6) it follows that $G_{2 r-1}^{x}$ is convex on $(a,(a+b) / 2)$, and hence $G_{2 r-1}^{x}(t)<0$ for $t \in(a,(a+b) / 2)$. Similarly, if $G_{2 r-3}^{x}(t)<0$ for $t \in(a,(a+b) / 2)$, then from (2.6) it follows that $G_{2 r-1}^{x}$ is concave on $(a,(a+b) / 2)$, and hence $G_{2 r-1}^{x}(t)>0$ for $t \in(a,(a+b) / 2)$. Since $G_{3}^{x}(t)<0$ for $t \in(a,(a+b) / 2)$, we can conclude that

$$
(-1)^{r-1} G_{2 r-1}^{x}(t)>0, \quad t \in(a,(a+b) / 2) .
$$

For the special cases $x=(a+b) / 2-(b-a) /(4 \sqrt{6})$ and $x=(a+b) / 2$, the proof is similar so we skip the details.

COROLLARY 2.5. For $r \geq 2$ and $x \in[a,(a+b) / 2-(b-a) /(4 \sqrt{6})]$, the functions $(-1)^{r} F_{2 r}^{x}(t)$ and $(-1)^{r} G_{2 r}^{x}(t)$ are strictly increasing on the interval $(a,(a+b) / 2)$ and strictly decreasing on the interval $((a+b) / 2, b)$. Consequently, $a$ and $b$ are the only zeros of $F_{2 r}^{x}$ in the interval $[a, b]$ and

$$
\begin{aligned}
& \max _{t \in[a, b]}\left|F_{2 r}^{x}(t)\right|=2\left|B_{2 r}\left(\frac{1}{2}-\frac{x-a}{b-a}\right)-B_{2 r}\left(\frac{x-a}{b-a}\right)+2\left(2^{-2 r}-1\right) B_{2 r}\right|, \\
& \max _{r \in[a, b]}\left|G_{2 r}^{x}(t)\right|=\left\{2\left|B_{2 r}\left(\frac{x-a}{b-a}\right)+B_{2 r}\right|, 2\left|B_{2 r}\left(\frac{1}{2}-\frac{x-a}{b-a}\right)+B_{2 r}\left(\frac{1}{2}\right)\right|\right\} .
\end{aligned}
$$

PROOF. Let $r \geq 2$ and $x \in[a,(a+b) / 2-(b-a) /(4 \sqrt{6}))$. We know that

$$
\left[(-1)^{r} F_{2 r}^{x}(t)\right]^{\prime}=\left[(-1)^{r} G_{2 r}^{x}(t)\right]^{\prime}=\frac{2 r}{b-a}(-1)^{r-1} G_{2 r-1}^{x}(t),
$$


and by Lemma 2.4 we also know that $(-1)^{r-1} G_{2 r-1}^{x}(t)>0$ for all $t \in(a,(a+b) / 2)$. Thus the functions $(-1)^{r} F_{2 r}^{x}(t)$ and $(-1)^{r} G_{2 r}^{x}(t)$ are strictly increasing on the interval $(a,(a+b) / 2)$. Also, by Lemma 2.2, we have $F_{2 r}^{x}(b-t)=F_{2 r}^{x}(t)$ and $G_{2 r}^{x}(b-t)=$ $G_{2 r}^{x}(t)$ for $t \in[a, b]$, which implies that $(-1)^{r} F_{2 r}^{x}(t)$ and $(-1)^{r} G_{2 r}^{x}(t)$ are strictly decreasing on the interval $((a+b) / 2, b)$. Further, $F_{2 r}^{x}(a)=F_{2 r}^{x}(b)=0$, which implies that $\left|F_{2 r}^{x}(t)\right|$ achieves its maximum at $t=(a+b) / 2$, that is,

$$
\begin{aligned}
\max _{r \in[a, b]}\left|F_{2 r}^{x}(t)\right| & =\left|F_{2 r}^{x}\left(\frac{a+b}{2}\right)\right| \\
& =2\left|B_{2 r}\left(\frac{1}{2}-\frac{x-a}{b-a}\right)-B_{2 r}\left(\frac{x-a}{b-a}\right)+2\left(2^{-2 r}-1\right) B_{2 r}\right| .
\end{aligned}
$$

Also,

$$
\begin{aligned}
\max _{t \in[a, b]}\left|G_{2 r}^{x}(t)\right| & =\max \left\{\left|G_{2 r}^{x}(a)\right|,\left|G_{2 r}^{x}\left(\frac{a+b}{2}\right)\right|\right\} \\
& =\max \left\{2\left|B_{2 r}\left(\frac{x-a}{b-a}\right)+B_{2 r}\right|, 2\left|B_{2 r}\left(\frac{1}{2}-\frac{x-a}{b-a}\right)+B_{2 r}\left(\frac{1}{2}\right)\right|\right\} .
\end{aligned}
$$

The special case $x=(a+b) / 2-(b-a) /(4 \sqrt{6})$ can be investigated similarly.

COROLlARY 2.6. For $r \geq 2$ the functions $(-1)^{r} F_{2 r}^{(a+b) / 2}(t)$ and $(-1)^{r} G_{2 r}^{(a+b) / 2}(t)$ are strictly increasing on the intervals $(a,(3 a+b) / 4)$ and $((a+b) / 2,(3 a+b) / 4)$, and strictly decreasing on the intervals $((3 a+b) / 4,(a+b) / 2)$ and $((3 a+b) / 4, b)$. Consequently, $a,(a+b) / 2$ and $b$ are the only zeros of $F_{2 r}^{(a+b) / 2}$ in the interval $[a, b]$ and

$$
\begin{aligned}
& \max _{r \in[a, b]}\left|F_{2 r}^{(a+b) / 2}(t)\right|=\left|F_{2 r}^{(a+b) / 2}((3 a+b) / 4)\right|=2^{2-2 r}\left(2-2^{1-2 r}\right)\left|B_{2 r}\right| \\
& \max _{r \in[a, b]}\left|G_{2 r}^{(a+b) / 2}(t)\right|=\left|G_{2 r}^{(a+b) / 2}((3 a+b) / 4)\right|=2^{2-2 r}\left(1-2^{1-2 r}\right)\left|B_{2 r}\right| .
\end{aligned}
$$

PROOF. The proof follows similarly to the proof of Corollary 2.5, using the fact that $F_{2 r}^{(a+b) / 2}((a+b) / 2)=2\left[B_{2 r}-B_{2 r}(1 / 2)+2\left(2^{-2 r}-1\right) B_{2 r}\right]=0$.

COROLlARY 2.7. For $r \geq 2$ and $x \in[a,(a+b) / 2-(b-a) /(4 \sqrt{6})]$, we have

$$
\begin{aligned}
\frac{1}{b-a} \int_{a}^{b}\left|F_{2 r-1}^{x}(t)\right| d t & =\frac{1}{b-a} \int_{a}^{b}\left|G_{2 r-1}^{x}(t)\right| d t=\frac{1}{r}\left|F_{2 r}^{x}\left(\frac{a+b}{2}\right)\right| \\
& =\frac{2}{r}\left|B_{2 r}\left(\frac{1}{2}-\frac{x-a}{b-a}\right)-B_{2 r}\left(\frac{x-a}{b-a}\right)+2\left(2^{-2 r}-1\right) B_{2 r}\right| .
\end{aligned}
$$

Also, we have

$$
\frac{1}{b-a} \int_{a}^{b}\left|F_{2 r}^{x}(t)\right| d t=2\left|B_{2 r}\left(\frac{x-a}{b-a}\right)+B_{2 r}\right|
$$


and

$$
\frac{1}{b-a} \int_{a}^{b}\left|G_{2 r}^{x}(t)\right| d t \leq 4\left|B_{2 r}\left(\frac{x-a}{b-a}\right)+B_{2 r}\right| .
$$

PROOF. Let $r \geq 2$ and $x \in[a,(a+b) / 2-(b-a) /(4 \sqrt{6})]$. Using Lemmas 2.2 and 2.4 we get

$$
\begin{aligned}
\int_{a}^{b}\left|G_{2 r-1}^{x}(t)\right| d t & =2\left|\int_{a}^{(a+b) / 2} G_{2 r-1}^{x}(t) d t\right| \\
& =2\left|-\frac{b-a}{2 r} G_{2 r}^{x}(s)\right|_{a}^{(a+b) / 2}\left|=\frac{b-a}{r}\right| G_{2 r}^{x}\left(\frac{a+b}{2}\right)-G_{2 r}^{x}(a) \mid \\
& =\frac{b-a}{r} F_{2 r}^{x}\left(\frac{a+b}{2}\right)
\end{aligned}
$$

which proves the first assertion. Using Corollary 2.5 and the fact that $F_{2 r}^{x}(a)=$ $F_{2 r}^{x}(b)=0$, we can deduce that the function $F_{2 r}^{x}$ does not change its sign on the interval $(a, b)$. Therefore we have

$$
\begin{aligned}
\int_{a}^{b}\left|F_{2 r}^{x}(t)\right| d t & =\left|\int_{a}^{b} F_{2 r}^{x}(t) d t\right|=\left|\int_{a}^{b}\left[G_{2 r}^{x}(t)-\widetilde{B}_{2 r}^{x}\right] d t\right| \\
& =\left|-\frac{b-a}{2 r+1} G_{2 r+1}^{x}(t)\right|_{a}^{b}-(b-a) \widetilde{B}_{2 r}^{x}|=(b-a)| \widetilde{B}_{2 r}^{x} \mid \\
& =2(b-a)\left|B_{2 r}\left(\frac{x-a}{b-a}\right)+B_{2 r}\right|
\end{aligned}
$$

which proves the second assertion. Finally, we use the triangle inequality to obtain the third formula.

Corollary 2.8. For $r \geq 2$, we have

$$
\int_{a}^{b}\left|F_{2 r-1}^{(a+b) / 2}(t)\right| d t=\int_{a}^{b}\left|G_{2 r-1}^{(a+b) / 2}(t)\right| d t=\frac{b-a}{r} 2^{4-2 r}\left(1-2^{-2 r}\right)\left|B_{2 r}\right| .
$$

Also,

$$
\frac{1}{b-a} \int_{a}^{b}\left|F_{2 r}^{(a+b) / 2}(t)\right| d t=2^{2-2 r}\left|B_{2 r}\right| \quad \text { and } \quad \frac{1}{b-a} \int_{a}^{b}\left|G_{2 r}^{(a+b) / 2}(t)\right| d t \leq 2^{3-2 r}\left|B_{2 r}\right| \text {. }
$$

PROOF. The proof is similar to the proof of Corollary 2.7.

LEMMA 2.9. Let $x \in[a,(a+b) / 2-(b-a) /(4 \sqrt{6})]$. If $f:[a, b] \rightarrow \mathbb{R}$ is such that for some $r \geq 2$ the derivative $f^{(2 r)}$ is continuous on $[a, b]$, then there exists a point $\eta \in[a, b]$ such that

$$
\widetilde{R}_{2 r}^{2}(x)=-\frac{(b-a)^{2 r}}{2(2 r) !}\left[B_{2 r}\left(\frac{x-a}{b-a}\right)+B_{2 r}\right] f^{(2 r)}(\eta) .
$$


ProOF. Let $x \in[a,(a+b) / 2-(b-a) /(4 \sqrt{6})]$. For $n=2 r \geq 4$ and $f$ such that $f^{(2 r)}$ is continuous on $[a, b]$ we can rewrite $R_{2 r}^{2}(f)$ as

$$
\widetilde{R}_{2 r}^{2}(x)=(-1)^{r} \frac{(b-a)^{2 r-1}}{4(2 r) !} \int_{a}^{b}(-1)^{r} F_{2 r}^{x}(t) f^{(2 r)}(t) d t=(-1)^{r} \frac{(b-a)^{2 r-1}}{4(2 r) !} I_{r},
$$

where

$$
I_{r}=\int_{a}^{b}(-1)^{r} F_{2 r}^{x}(t) f^{(2 r)}(t) d t
$$

If $m=\min _{[a, b]} f^{(2 r)}(t)$ and $M=\max _{[a, b]} f^{(2 r)}(t)$, then $m \leq f^{(2 r)}(t) \leq M, t \in[a, b]$. From Corollary 2.5 we have $(-1)^{r} F_{2 r}^{x}(t) \geq 0, t \in[a, b]$, so

$$
m \int_{a}^{b}(-1)^{r} F_{2 r}^{x}(t) d t \leq I_{r} \leq M \int_{a}^{b}(-1)^{r} F_{2 r}^{x}(t) d t
$$

Since

we obtain

$$
\int_{a}^{b} F_{2 r}^{x}(t) d t=-(b-a) \widetilde{B}_{2 r}^{x}=-2(b-a)\left[B_{2 r}\left(\frac{x-a}{b-a}\right)+B_{2 r}\right],
$$

$$
\begin{aligned}
& 2 m(-1)^{r-1}(b-a)\left[B_{2 r}\left(\frac{x-a}{b-a}\right)+B_{2 r}\right] \\
& \quad \leq I_{r} \leq 2 M(-1)^{r-1}(b-a)\left[B_{2 r}\left(\frac{x-a}{b-a}\right)+B_{2 r}\right] .
\end{aligned}
$$

By the continuity of $f^{(2 r)}$ on $[a, b]$ it follows that there must exist a point $\eta \in[a, b]$ such that

$$
I_{r}=2(-1)^{r-1}(b-a)\left[B_{2 r}\left(\frac{x-a}{b-a}\right)+B_{2 r}\right] f^{(2 r)}(\eta) .
$$

From that we can easily obtain (2.7).

LEMMA 2.10. If $f:[a, b] \rightarrow \mathbb{R}$ is such that for some $r \geq 2$ the derivative $f^{(2 r)}$ is continuous on $[a, b]$, then there exists a point $\eta \in[a, b]$ such that

$$
\widetilde{R}_{2 r}^{2}\left(\frac{a+b}{2}\right)=-\frac{(b-a)^{2 r}}{(2 r) !} 2^{-2 r} B_{2 r} f^{(2 r)}(\eta) .
$$

PROOF. The proof follows analogously to the proof of Lemma 2.9.

THEOREM 2.11. Let $x \in[a,(a+b) / 2-(b-a) /(4 \sqrt{6})]$. Assume that $f:[a, b] \rightarrow \mathbb{R}$ is such that $f^{(2 r)}$ is continuous on $[a, b]$ for some $r \geq 2$. If $f$ is a $(2 r)$-convex or (2r)-concave function, then there exists a point $\vartheta \in[0,1]$ such that

$$
\begin{aligned}
\widetilde{R}_{2 r}^{2}(x)= & \vartheta\left[B_{2 r}\left(\frac{1}{2}-\frac{x-a}{b-a}\right)-B_{2 r}\left(\frac{x-a}{b-a}\right)+2\left(2^{-2 r}-1\right) B_{2 r}\right] \\
& \times \frac{(b-a)^{2 r-1}}{2(2 r) !}\left[f^{(2 r-1)}(b)-f^{(2 r-1)}(a)\right] .
\end{aligned}
$$


Proof. By Corollary 2.5 for $t \in[a, b]$ we have

$$
0 \leq(-1)^{r-1} F_{2 r}^{x}(t) \leq(-1)^{r-1} F_{2 r}^{x}((a+b) / 2) .
$$

The rest of the proof is similar to the proof of Lemma 2.9.

Theorem 2.11 can be improved in a way that the derivative $f^{(2 r)}$ need not be continuous on $[a, b]$. To obtain such a result we use the following theorem from [7, Theorem 1].

THEOREM B. Let $\varphi: I \rightarrow \mathbb{R}, I \subset \mathbb{R}$, be a monotonic function, and let $\rho:$ $\mathbb{R} \rightarrow \mathbb{R}$ be a periodic function with period $P$ such that for some $a \in \mathbb{R}$ and $n \in \mathbb{N}$ $[a, a+n P] \subset I$. Suppose that there exists some $x_{0} \in(a, a+P)$ such that $\rho\left(x_{0}\right)=0$, $\rho(x) \geq 0$ for all $x \in\left[a, x_{0}\right)$ and $\rho(x) \leq 0$ for all $x \in\left(x_{0}, a+P\right]$. Suppose also that $\int_{a}^{a+P} \rho(x) d x=0$. If $\varphi$ is increasing on $[a, a+n P]$, then

$$
-\int_{a}^{a+n P} \rho(x) \varphi(x) d x \leq \frac{1}{2 n}(\varphi(a+n P)-\varphi(a)) \int_{a}^{a+n P}|\rho(x)| d x,
$$

and this inequality is sharp. If $\varphi$ is decreasing on $[a, a+n P]$, then the inequality (2.8) is reversed.

THEOREM 2.12. Assume that the function $f:[a, b] \rightarrow \mathbb{B}$ is such that for some $r \geq 2$ the derivative $f^{(2 r-1)}$ is continuous and increasing on $[a, b]$. Then for every $x \in[a,(a+b) / 2-(b-a) /(4 \sqrt{6})]$ we have

$$
\begin{aligned}
(-1)^{r} & \left\{\frac{1}{b-a} \int_{a}^{b} f(t) d t-\frac{f(a)+f(b)+f(x)+f(a+b-x)}{4}+\widetilde{T}_{2 r-1}(x)\right\} \\
\leq & \frac{(b-a)^{2 r-1}}{2(2 r) !}\left[f^{(2 r-1)}(b)-f^{(2 r-1)}(a)\right] \\
& \times\left|B_{2 r}\left(\frac{1}{2}-\frac{x-a}{b-a}\right)-B_{2 r}\left(\frac{x-a}{b-a}\right)+2\left(2^{-2 r}-1\right) B_{2 r}\right|,
\end{aligned}
$$

and this inequality is sharp.

Proof. We know that the function $F_{2 r-1}^{x}$ is periodic with period $P=b-a$. From Theorem 2.4 and Lemma 2.2 for $r \geq 2$ and $x \in[a,(a+b) / 2-(b-a) /(4 \sqrt{6})]$ we have: $F_{2 r-1}^{x}((a+b) / 2)=0, \int_{a}^{b} F_{2 r-1}^{x}(t) d t=0$ and also

$$
(-1)^{r-1} F_{2 r-1}^{x}(t) \begin{cases}>0, & t \in(a,(a+b) / 2), \\ <0, & t \in((a+b) / 2, b) .\end{cases}
$$


This means that if in Theorem B we choose $\rho(t)=(-1)^{r-1} F_{2 r-1}^{x}(t), \varphi(t)=f^{(2 r-1)}(t)$ and $n=1$, then from (2.8) we obtain

$$
-\int_{a}^{b}(-1)^{r-1} F_{2 r-1}^{x}(t) f^{(2 r-1)}(t) d t \leq \frac{1}{2}\left[f^{(2 r-1)}(b)-f^{(2 r-1)}(a)\right] \int_{a}^{b}\left|F_{2 r-1}^{x}(t)\right| d t
$$

and combining this with Corollary 2.7 we obtain

$$
\begin{aligned}
(-1)^{r} \int_{a}^{b} F_{2 r-1}^{x}(t) f^{(2 r-1)}(t) d t & \leq \frac{b-a}{r}\left[f^{(2 r-1)}(b)-f^{(2 r-1)}(a)\right] \\
& \times\left|B_{2 r}\left(\frac{1}{2}-\frac{x-a}{b-a}\right)-B_{2 r}\left(\frac{x-a}{b-a}\right)+2\left(2^{-2 r}-1\right) B_{2 r}\right|
\end{aligned}
$$

From Theorem 2.1 we know that

$$
\begin{aligned}
& \frac{1}{b-a} \int_{a}^{b} f(t) d t-\frac{f(a)+f(b)+f(x)+f(a+b-x)}{4}+\widetilde{T}_{2 r-1}(x) \\
& \quad=\frac{(b-a)^{2 r-2}}{4(2 r-1) !} \int_{[a, b]} F_{2 r-1}^{x}(t) f^{(2 r-1)}(t) d t
\end{aligned}
$$

so

$$
\begin{aligned}
(-1)^{r} & \left\{\frac{1}{b-a} \int_{a}^{b} f(t) d t-\frac{f(a)+f(b)+f(x)+f(a+b-x)}{4}+\widetilde{T}_{2 r-1}(x)\right\} \\
= & \frac{(b-a)^{2 r-2}}{4(2 r-1) !}(-1)^{r} \int_{a}^{b} F_{2 r-1}^{x}(t) f^{(2 r-1)}(t) d t \\
\leq & \frac{(b-a)^{2 r-1}}{2(2 r) !}\left[f^{(2 r-1)}(b)-f^{(2 r-1)}(a)\right] \\
& \times\left|B_{2 r}\left(\frac{1}{2}-\frac{x-a}{b-a}\right)-B_{2 r}\left(\frac{x-a}{b-a}\right)+2\left(2^{-2 r}-1\right) B_{2 r}\right|
\end{aligned}
$$

THEOREM 2.13. Assume that the function $f:[a, b] \rightarrow \mathbb{R}$ is such that for some $r \geq 2$ the derivative $f^{(2 r-1)}$ is continuous and increasing on $[a, b]$. Then we have

$$
\begin{aligned}
& (-1)^{r}\left\{\frac{1}{b-a} \int_{a}^{b} f(t) d t-\frac{f(a)+f(b)+2 f((a+b) / 2)}{4}+\widetilde{T}_{2 r-1}\left(\frac{a+b}{2}\right)\right\} \\
& \leq \frac{(b-a)^{2 r-1}}{(2 r) !}\left[f^{(2 r-1)}(b)-f^{(2 r-1)}(a)\right] 2^{1-2 r}\left(1-2^{-2 r}\right)\left|B_{2 r}\right|,
\end{aligned}
$$

and this inequality is sharp.

Proof. The proof is similar to the proof of Theorem 2.12 . 


\section{Hadamard's inequalities for ( $2 r$-convex functions}

Now we can give our main result: a generalization of Hadamard's inequalities for (2r)-convex functions, $r \geq 2$.

THEOREM 3.1. Assume that $f:[a, b] \rightarrow \mathbb{R}$ is such that for some $r \geq 2$ the derivative $f^{(2 r-1)}$ is continuous on $[a, b]$, and assume that $f$ is $(2 r)$-convex on $[a, b]$. If $r$ is odd, then for all $x \in[a,(a+b) / 2-(b-a) /(4 \sqrt{6})] \cup\{(a+b) / 2\}$

$$
\begin{aligned}
& \frac{f(a)+f(b)}{2}-\frac{1}{b-a} \int_{a}^{b} f(t) d t-\widetilde{T}_{2 r-1}^{F}, \\
& \quad \geq \frac{1}{b-a} \int_{a}^{b} f(t) d t-\frac{f(x)+f(a+b-x)}{2}+\widetilde{T}_{2 r-1}^{V}(x),
\end{aligned}
$$

and for all $x \in[a+(b-a) /(2 \sqrt{3}),(a+b) / 2]$

$$
\frac{1}{b-a} \int_{a}^{b} f(t) d t-\frac{f(x)+f(a+b-x)}{2}+\widetilde{T}_{2 r-1}^{v}(x) \geq 0 .
$$

If $r$ is even the above inequalities are reversed.

Proof. Let $x \in[a,(a+b) / 2-(b-a) /(4 \sqrt{6})]$. In the case $n=2 r \geq 4$, from (2.5) we get

$$
\frac{2}{b-a} \int_{a}^{b} f(t) d t-\frac{f(a)+f(b)+f(x)+f(a+b-x)}{2}+2 \widetilde{T}_{2 r-1}(x)=2 \widetilde{R}_{2 r}^{2}(f),
$$

where

$$
\widetilde{R}_{2 r}^{2}(x)=\frac{(b-a)^{2 r-1}}{4(2 r) !} \int_{[a, b]} F_{2 r}^{x}(t) d f^{(2 r-1)}(t) .
$$

If $f$ is $(2 r)$-convex then $d f^{(2 r-1)}(t) \geq 0$ on $[a, b]$, and since by Corollary 2.5 we know that $(-1)^{r} F_{2 r}^{x}(t) \geq 0, t \in[a, b]$, we obtain $\widetilde{R}_{2 r}^{2}(x) \geq 0$ for $r$ even and $\widetilde{R}_{2 r}^{2}(x) \leq 0$ for $r$ odd. The same is true if $x=(a+b) / 2$. This means that for $r$ odd we have

$$
\frac{2}{b-a} \int_{a}^{b} f(t) d t-\frac{f(a)+f(b)+f(x)+f(a+b-x)}{2}+2 \widetilde{T}_{2 r-1}(x) \leq 0
$$

that is,

$$
\begin{aligned}
& \frac{f(a)+f(b)}{2}-\frac{1}{b-a} \int_{a}^{b} f(t) d t-\widetilde{T}_{2 r-1}^{F} \\
& \quad \geq \frac{1}{b-a} \int_{a}^{b} f(t) d t-\frac{f(x)+f(a+b-x)}{2}+\widetilde{T}_{2 r-1}^{v}(x),
\end{aligned}
$$


and the above inequality is reversed if $r$ is even. This completes the proof of (3.1).

Now let $x \in[a+(b-a) /(2 \sqrt{3}),(a+b) / 2]$ and suppose that $r$ is odd. We can use the analogous results from [9, Theorem 2.1 and Corollary 2.4] to obtain

$$
\frac{1}{b-a} \int_{a}^{b} f(t) d t-\frac{f(x)+f(a+b-x)}{2}+\widetilde{T}_{2 r-1}^{v}(x) \geq 0,
$$

and the reverse if $r$ is even. This completes the proof.

The interested reader can find several sharper variants of (3.2) in [8].

\section{References}

[1] M. Abramowitz and I. A. Stegun (eds.), Handbook of mathematical functions with formulae, graphs and mathematical tables, Applied Math. Series 55, 4th printing (National Bureau of Standards, Washington, 1965).

[2] I. S. Berezin and N. P. Zhidkov, Computing Methods, Vol. I (Pergamon Press, Oxford, 1965).

[3] P. S. Bullen, "Error estimates for some elementary quadrature rules", Univ. Beograd Publ. Elektrotehn. Fak., Ser. Mat. Fiz. 602-633 (1978) 97-103.

[4] Lj. Dedić, M. Matić and J. Pečarić, "On generalizations of Ostrowski inequality via some Euler-type identities”, Math. Inequal. Appl. 3 (2000) 337-353.

[5] Lj. Dedić, M. Matić and J. Pečarić, "On Euler trapezoid rule", Appl. Math. Comput. 123 (2001) $37-62$.

[6] Lj. Dedić, M. Matić, J. Pečarić and A. Vukelić, "Hadamard-type inequalities via some Euler-type identities-Euler bitrapezoid formulae”, Nonlinear Stud. 8 (2001) 343-372.

[7] M. Klaričić Bakula, J. Pečarić and A. Vukelić, "Interpolation of periodic functions and applications on some integration formulae of interpolatory type", Bull. Math. Soc. Sci. Math. Roumanie 48 (2005) 261-275.

[8] J. Pečarić and A. Vukelić, Hadamard and Dragomir-Agarwal inequalities, the Euler formulae and convex functions, Functional Equations, Inequalities and Applications (Kluwer, Dordrecht, 2003).

[9] J. E. Pečarić, I. Perić and A. Vukelić, "Sharp integral inequalities based on general Euler two-point formulae", ANZIAM J. 46 (2005) 555-574.

[10] J. E. Pečarić, F. Proschan and Y. L. Tong, Convex functions, partial orderings, and statistical applications (Academic Press, New York, 1992).

[11] A. W. Roberts and D. E. Varberg, Convex Functions (Academic Press, New York, 1973). 